\title{
The INTEGRAL Complete Sample of Type 1 AGN
}

\author{
M. Molina*, on behalf of the INTEGRAL AGN Team \\ INAF/IASF Milano, Italy \\ E-mail: molina@iasf-milano.inaf.it
}

We present the broad-band X-ray characteristics of a complete sample of 36 type 1 AGN, detected by INTEGRAL in the 20-40 keV band above the $5.5 \sigma$ level. We performed the broad-band (1-110 $\mathrm{keV}$ ) spectral analysis using INTEGRAL/Swift/BAT observations together with XMM-Newton, Chandra, ASCA and Swift/XRT data. We also present the general average properties of the sample, i.e. the distribution of photon indices, high energy cut-offs, reflection fractions and absorption properties, together with an in-depth analysis of their parameter space. We find that the average Seyfert 1 power law has an index of 1.7 with a dispersion of 0.2 . The mean cut-off energy is at around $100 \mathrm{keV}$, with most objects displaying $\mathrm{E}_{c}$ in the range $50-150 \mathrm{keV}$; the average amount of Compton reflection is 1.5 with a typical dispersion of 0.7 . We do not find any convincing correlation between the various parameters, an indication that our analysis is not strongly dependent by the interplay between them. Finally, we investigate how the results presented in this work fit into current frameworks for AGN spectral modeling and Cosmic Diffuse X-ray Background synthesis models.

The Extreme sky: Sampling the Universe above $10 \mathrm{keV}$

October 13-17 2009

Otranto (Lecce) Italy

${ }^{*}$ Speaker. 


\section{Introduction}

It is now widely accepted that the Cosmic X-ray Background (CXB) is the product of the integrated emission of point-like extragalactic sources, most of which are AGN [2], as predicted by AGN synthesis models [5]. However, while below $10 \mathrm{keV}$ the shape and the intensity of the CXB is quite well understood [17], at higher energies, around $30 \mathrm{keV}$ or above, these quantities are less well studied and the AGN contribution less defined. Recent analyses have allowed a better estimate of the CXB spectrum above $10 \mathrm{keV} \mathrm{[1];} \mathrm{these} \mathrm{measurements} \mathrm{agree} \mathrm{with} \mathrm{an} \mathrm{early} \mathrm{HEAO} \mathrm{A1} \mathrm{result}$ [15] and are consistent within their systematic uncertainties. Such consistency is not present with data at lower energies, where the measured spectrum may be up to $\sim 40 \%$ larger. Synthesis models need now to take this discrepancy into consideration. Parameters like the covering fraction and the geometry of the Comptonising plasma are also needed to reproduce the shape of the CXB above $30 \mathrm{keV}$ [6]. The fraction of Compton Thick sources and the high energy cut-off of the primary continuum emission have to be considered as well. Some recent models [13, 12] also propose that the power law slope, and its spread, and the reflection component may be important parameters for models of the CXB. Determining all these parameters up to $100 \mathrm{keV}$ and beyond is important so to obtain a firm description of the AGN contribution to the CXB and hence a better understanding of the accretion history of the Universe.

The continuum power law slope and its high energy cut-off are also essential for AGN spectral modelling, since they are linked to the characteristics of the Comptonising region around the central nucleus. These quantities are crucial for understanding models and discriminating between them. While the photon index distribution has been well investigated [31, 29], observational results on the cut-off energy have so far been limited by the scarcity of measurements above $10-20 \mathrm{keV}$.

Here we present the analysis on the INTEGRAL complete sample of 36 type 1 active galaxies, selected in the 20-40 keV band and listed in the third IBIS/ISGRI catalogue [4].

\section{The Complete Sample: General Properties}

The spectra of the 36 type 1 AGN have been fitted with an exponentially cut-off power law model, reflected from neutral material, plus Galactic and intrinsic absorption and a gaussian component to model the iron $\mathrm{K} \alpha$ line at $6.4 \mathrm{keV}$ when needed.

Almost $60 \%$ of the sample sources, despite being type $1 \mathrm{AGN}$, require absorption in excess of the Galactic value; for these absorbed objects, the majority (86\%) have a simple absorber fully covering the source, while $24 \%$ require (in addition or in substitution) a more complex type of absorption (see left panel of figure 1 for the $\mathrm{N}_{H}$ distribution). The presence of strong absorption up to $2-5 \times 10^{23} \mathrm{~cm}^{-2}$ in these AGN is at odds with their type 1 classification, but this can be explained assuming the "clumpy torus" model [9]. In this model, the torus is not a continuous toroidal structure, but is rather made of clouds with $10^{22}<\mathrm{N}_{H}<10^{23} \mathrm{~cm}^{-2}$, distributed around the equatorial plane of the AGN. Therefore, the difference between type 1 and 2 AGN is not only due to orientation but also on how many clouds our line of sight intercepts. The average value of the absorbing column density is $0.4 \times 10^{22} \mathrm{~cm}^{-2}$, with a larger or smaller spread depending on whether complex absorption objects are included or not. Our mean values are close to the average column density for broad line AGN observed by BAT [35]; much higher average values were instead found 

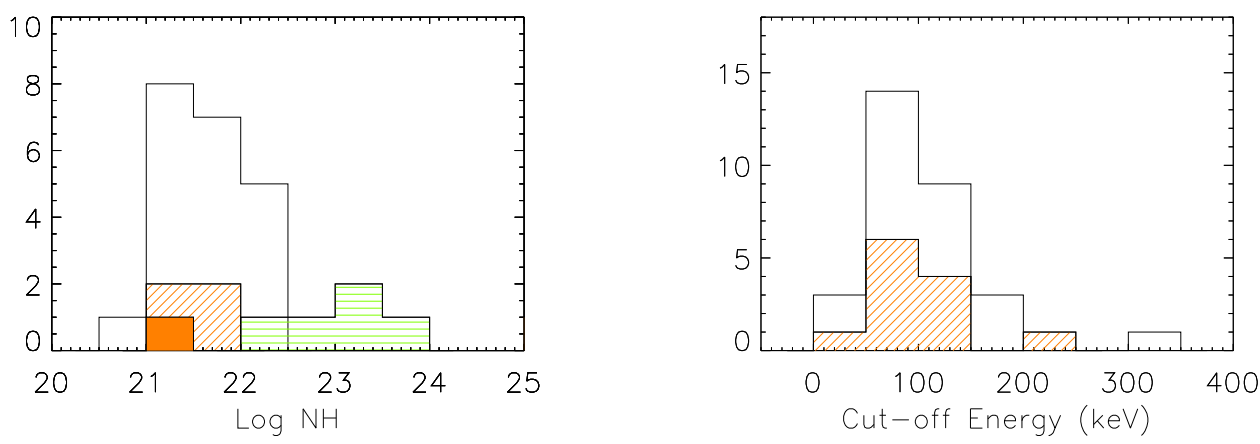

Figure 1: Left Panel: intrinsic $\mathrm{N}_{H}$ distribution (horizontally hatched histogram: complex absorption; diagonally hatched upper limits; filled histogram: lower limit) Right Panel: high energy cut-off distribution (hatched histogram: lower limits).

in other works $[8,25]$, possibly due to the presence of complex absorption sources in these samples. It is however difficult to compare results when different fitting models/approaches are used and care must be taken when making such comparisons.

The mean photon index for the whole sample is $1.74 \pm 0.2$, flatter than the canonical value of 1.9 generally assumed for type 1 AGN. It is also lower than the value found for 43 type 1 AGN observed with BeppoSAX [8], possibly because we made use of a complete sample of hard X-ray selected AGN, rather than randomly chosen bright sources. Other results [35, 25, 33], obtained using both soft and hard X-ray data for type 1 or for both type 1 and 2 AGN, provide similar values to what we find in our sample.

The mean high energy cut-off is $109 \pm 63 \mathrm{keV}$; the distribution of values for this parameter shows that most objects fall within the range 50 to $150 \mathrm{keV}$ (see right panel of figure 1). Our value is lower than the mean value of $230 \mathrm{keV}$ found for sources observed with BeppoSAX [8, 18]. The sample has also been tested against the correlation between the photon index and the high energy cut-off [30, 21, 28], but we found no evidence for it.

The mean reflection fraction is $1.5 \pm 0.7$; the parameter distribution has a peak at around with a tail extending to values of $R$ as high as 11.5. ${ }^{1}$ Such strong reflection might be present when more primary X-ray radiation is emitted toward the reflector than toward the observer, as is possible in the case of strongly variable nuclear emission or when there is a time delay between the underlying continuum and the reflected component [20,26]. Another explanation might be a peculiar geometry [19] or general relativistic light bending effects [10, 22, 11]. In other cases, the reflection fraction is very high simply because there is a large mismatch between X-ray and soft gamma ray data due to flux variability $[23,24]$.

We tested the reflection fraction and the cross-clibration constant for a correlation, since they are related in the fitting procedure, but found that, at most, a weak one exists. A correlation between the photon index and the reflection fraction was also investigated. This correlation might be explained assuming that the cold medium, responsible for the reflection and surrounding the

\footnotetext{
${ }^{1}$ These sources are not accounted for in the average calculation.
} 

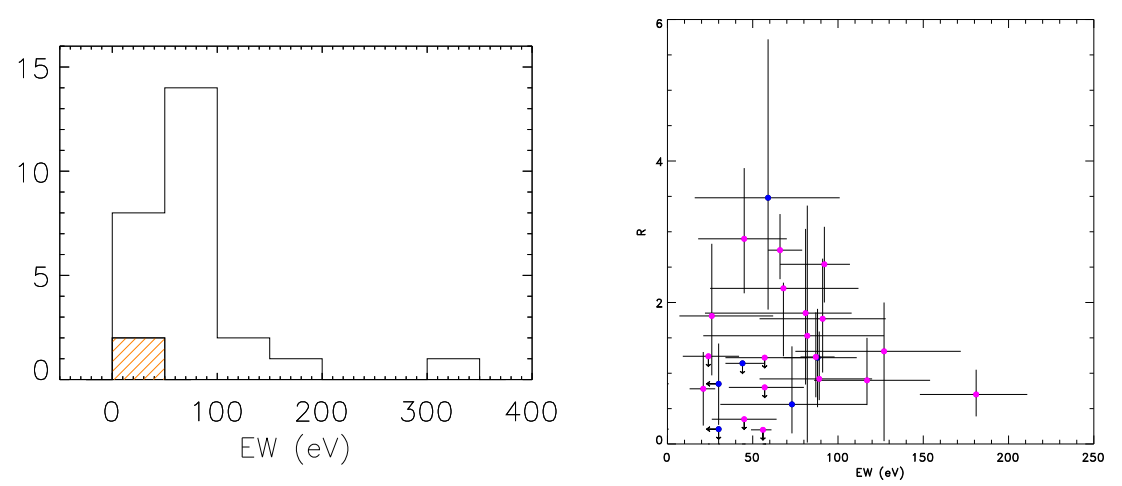

Figure 2: Left Panel: iron line EW distribution. (hatched histogram: upper limits). Right Panel: reflection fraction vs. EWfor radio-loud (blue boxes) and radio-quiet (magenta circles) sources.

corona, affects the hardness of the X-ray spectrum [36, 28]. However, it has to be noted that $R$ and $\Gamma$ might be strongly linked in the fitting procedure and therefore a trend in the parameter space may not be entirely physical. In our data there is no evidence for such a correlation. We have also looked for a correlation between the reflection fraction and the high energy cut-off, but found none. We can therefore conclude that we do not find any evidence for correlations between $\Gamma, R$ and $\mathrm{E}_{c}$, an indication that the interplay between these quantities does not have an important part in the fitting procedure and has not introduced spurious relationships in our analysis.

The distribution of the iron $K \alpha$ line equivalent widths is shown in left panel of figure 2. The mean value of EW is $83 \pm 61 \mathrm{eV}$, fully compatible with the results shown in [3]. Our value is lower than the average of $177 \mathrm{keV}$ found by [35] using only the type 1 AGN in their sample; their spread is however large $(142 \mathrm{eV})$ so that our result overlaps theirs. We also found that most sources have narrow $\mathrm{Fe} \mathrm{K} \alpha$ lines, with only $\sim 15 \%$ of the sample requiring a broad line, lower than what found by [16]. We also searched the data for the anti-correlation between EW and source luminosity, the so-called "X-ray Baldwin" or "Iwasawa-Taniguchi" effect [20], but found none. If the iron line emission is entirely associated with the optically thick material of the disk, one would expect the line EW to correlate with the reflection fraction. Figure 2 (right panel) shows a plot of $R$ vs. EW for the entire sample, with radio loud objects separated from radio quiet sources; as can be seen, there is an indication of a weak correlation at most. Note that the reprocessing features in BLRG tend to be, on average, quite weak with respect to their radio quiet counterparts $[14,32]$.

\section{The Complete Sample: Spectral Modeling}

A good fraction of AGN spectral models ascribe the power law to the inverse Compton scattering of soft photons off hot electrons. Within this scenario, the power law photon index and the high energy cut-off are directly linked to the temperature and optical depth of the Comptonising hot plasma. Knowing these two quantities is therefore very important for understanding the characteristics of the plasma near the central engine. The values of $\Gamma$ and $\mathrm{E}_{c}$ are in fact linked to the Comptonising hot plasma temperature $\mathrm{kT}_{e}$ and optical depth $\tau$ by a simple relation [28] In our 
sample the mean value of $\mathrm{E}_{c}$ is $\sim 110 \mathrm{keV}$, with most objects in the sample falling in the range $50-150 \mathrm{keV}$, hence suggesting a range of plasma temperatures from 20 to $80 \mathrm{keV}$ (or $2-9 \times 10^{8} \mathrm{~K}$ ). As for the optical depth of the plasma, with our results we find $\tau$ in the range 1 to 4 . These values are in agreement with previous results for a sample of Seyfert 1s [28].

\section{The Complete Sample: Cosmic Diffuse X-ray Background}

A fundamental parameter for CXB synthesis models is the broad-band spectral shape of type 1 AGN. The average slope of the power law is generally taken to be $\Gamma=1.9$, mainly from observations performed at low energies; a harder average spectrum means a stronger contribution from unabsorbed AGN and hence a lower number of obscured sources required to match the CXB. Up to now the dispersion around this mean photon index value has generally been neglected, but recently [13] have shown that the CXB spectrum can be hardened near the $30 \mathrm{keV}$ peak by as much as $20-30 \%$ by taking into account a dispersion in photon indices of 0.2 or more. Since observations above $10 \mathrm{keV}$ have been less frequent up to now, the cut-off energy and the reflection bump are the less well constrained parameters. While it is well known that an exponential cut-off must be present around a few hundreds of $\mathrm{keV}$, in order not to violate the present level of the CXB above $100 \mathrm{keV}$, values of $200 \mathrm{keV}$ and up to $500 \mathrm{keV}$ have been used in the past. A better constraint on $\mathrm{E}_{c}$ is therefore a very important issue. The characteristic hard slope and the $40 \mathrm{keV}$ break of the CXB indicate, besides the existence of heavily absorbed objects, the presence of at least some reflection in AGN spectral energy distributions, and CXB synthesis models typically use a reflection fraction $\mathrm{R} \sim 1$ [13]; [12]. Clearly the higher the reflection, the less important becomes the contribution of Compton thick AGN.

Taking all these facts into account, it can be stated that the CXB is simply the integrated emission of absorbed and unabsorbed AGN, as shown by the cumulative X-ray spectrum of local AGN selected at high energies [35], [33], [34].

Our data focused for the first time on a high energy complete sample of type 1 AGN might be able to provide key information on the AGN average continuum slope and dispersion and a better definition of $R$ and $\mathrm{E}_{c}$. In the recent work of [13], a $\Gamma$ of 1.9, a cut-off energy of $200 \mathrm{keV}$ and a reflection of 1 were assumed, while the spectral index dispersion was similar to the value found in the present work. A flatter $\Gamma$ and a lower $\mathrm{E}_{c}$ for the average spectrum of unabsorbed sources have a strong impact on the synthesis model of the $\mathrm{CXB}$, particularly with respect to the fraction of Compton thick sources required to match the $30 \mathrm{keV}$ peak. Although our results are in contrast with previous studies, we note that they agree with the recent estimate of the cumulative hard X-ray spectrum of local INTEGRAL AGN [33, 35]. Thus the observational evidence gathered so far by hard X-ray surveys points to a flatter average spectrum and possibly a lower $\mathrm{E}_{c}$ as found in this work. It would be interesting to explore the consequences of fitting the CXB with our range of parameters, particularly with reference to the fraction of Compton thick sources needed to provide a self-consistent fit.

\section{References}

[1] Ajello M., Rau A., Greiner J. et al. 2008, ApJ, 673, 96 
[2] Barger A.J., Cowie L.L., Mushotzky R.F. et al. 2005, AJ, 129, 578

[3] Bianchi S., Guainazzi M., Matt G. et al. 2009, A\&A, 495, 421

[4] Bird A. J., Malizia A., Bazzano A. et al. 2007, ApJS, 170, 175

[5] Comastri A., Setti G., Zamorani G. \& Hasinger G. 1995, A\&A, 296, 1

[6] Comastri A., Gilli R. \& Hasinger G. 2006, “Gamma Wave 2005”, Bonifacio, September 2005.

[7] Dadina M. 2007, A\&A, 461, 1209

[8] Dadina M. 2008, A\&A, 485, 417

[9] Elitzur M. \& Shlosman I. 2006, ApJ, 648, 101

[10] Fabian A.C., Miniutti G., Gallo L. et al. 2004, MNRAS, 353, 1071

[11] Fabian A.C., Miniutti G., Iwasawa K., \& Ross R.R. 2005, MNRAS, 361, 795

[12] Gandhi P., Fabian A.C., Suebsuwong T. et al. 2007, MNRAS, 382, 1005

[13] Gilli R., Comastri A. \& Hasinger G. 2007, A\&A, 463, 79

[14] Grandi P., Malaguti G. \& Fiocchi M. 2006, ApJ, 642, 113

[15] Gruber D.E., Matteson J.L., Peterson L. E. \& Jung G. V. 1999, ApJ, 520, 124

[16] Guainazzi M., Bianchi S. \& Dovčiak M. 2006, AN, 327, 1032

[17] Hickox R.C. \& Markevitch M. 2006, ApJ, 645, 95

[18] Malizia A., Bassani L., Stephen J.B. et al. 2003, ApJ, 589, 17

[19] Malzac J., Beloborodov A. M. \& Poutanen J. 2001, MNRAS, 326, 417

[20] Malzac J. \& Petrucci P.O. 2002, MNRAS, 336, 1209

[21] Matt G. 2001, AIPC, 599, 209

[22] Miniutti G. \& Fabian A.C. 2004, MNRAS, 349, 1435

[23] Molina M., Malizia A., Bassani L. et al. 2006, MNRAS, 371, 821

[24] Molina M., Bassani L., Malizia A. et al. 2008, MNRAS, 390, 1217

[25] Mushtozky R. 1984, Adv. Space Res. Vol.3, N10-12, 157

[26] Panessa F., Bassani L., De Rosa A. et al. 2008, A\&A, 483, 151

[27] Perola G.C., Matt G., Cappi M. et al. 2002, A\&A, 389, 802

[28] Petrucci P.O., Haardt F., Maraschi L. et al. 2001, ApJ, 556, 716

[29] Piconcelli E., Jiménez-Bailón E., Guainazzi M. et al. 2005, A\&A, 432, 15

[30] Piro L. 1999, AN, 320, 236

[31] Reeves J. N. \& Turner, M. J. L. 2000, MNRAS, 316, 234

[32] Sambruna R.M., Eracleous M., Mushotzky R.F. 1999, ApJ, 526, 60

[33] Sazonov S., Krivonos R., Revnivtsev M. et al. 2008, A\&A, 482, 517

[34] Triester E., Urry C.M., Virani S. 2009, ApJ, 696, 110

[35] Winter L.M., Mushotzky R.F., Reynolds C.S. \& Tueller J. 2009, ApJ, 690, 1322

[36] Zdziarski A.A., Ghisellini G., George I.M. et al. 1990, ApJ, 363, 1 